\title{
A genetic risk score to guide age-specific, personalized prostate cancer screening
}

Tyler M. Seibert, MD ${ }^{1,2}$

Chun Chieh Fan, $\mathrm{MD}^{1,3}$

Yunpeng Wang, $\mathrm{PhD}^{4}$

Verena Zuber, $\mathrm{PhD}^{4}$

Roshan Karunamuni, $\mathrm{PhD}^{1,2}$

J. Kellogg Parsons, $M^{5}$

Rosalind A. Eeles, $\mathrm{PhD}^{6,7}$

Douglas F. Easton, $\mathrm{PhD}^{8}$

ZSofia Kote-Jarai, $\mathrm{PhD}^{6}$

Ali Amin Al Olama, $\mathrm{PhD}^{8}$

Sara Benlloch Garcia, $\mathrm{PhD}^{8}$

Kenneth Muir, $\mathrm{PhD}^{9,10}$

Henrik Gronberg, Prof ${ }^{11}$

Fredrik Wiklund, $\mathrm{PhD}^{11}$

Markus Aly, PhD ${ }^{11,12}$

Johanna Schleutker, $\mathrm{PhD}^{13,14}$

Csilla Sipeky, $\mathrm{PhD}^{15}$

Teuvo LJ Tammela, Prof ${ }^{16}$

Børge G. Nordestgaard, Prof ${ }^{17,18}$

Sune F. Nielsen, $\mathrm{PhD}^{17,18}$

Maren Weischer, $\mathrm{MD}^{18}$

Rasmus Bisbjerg, MD ${ }^{19}$

M. Andreas Røder, $\mathrm{MD}^{20}$

Peter Iversen, Prof ${ }^{17,20}$

Tim J. Key, DPhil ${ }^{21}$

Ruth C. Travis, DPhil ${ }^{21}$

David E. Neal, FMedSci ${ }^{22,23}$

Jenny L. Donovan, $\mathrm{PhD}^{24}$

Freddie C. Hamdy, FMedSci ${ }^{25}$

Paul Pharoah, Prof $^{26}$

Nora Pashayan, MD ${ }^{27,26}$

Kay-Tee Khaw, FRCP ${ }^{28}$

Christiane Maier, $\mathrm{PhD}^{29}$

Walther Vogel, Prof $^{29}$

Manuel Luedeke, $\mathrm{PhD}^{29}$

Kathleen Herkommer, MD $^{30}$

Adam S. Kibel, MD ${ }^{31}$

Cezary Cybulski, MD ${ }^{32}$

Dominika Wokolorczyk, $\mathrm{PhD}^{32}$

Wojciech Kluzniak, MS ${ }^{32}$

Lisa Cannon-Albright, $\mathrm{PhD}^{33,34}$

Hermann Brenner, Prof ${ }^{35,36,37}$ 
Katarina Cuk, $\mathrm{PhD}^{35}$

Kai-Uwe Saum, $\mathrm{PhD}^{35}$

Jong Y. Park, $\mathrm{PhD}^{38}$

Thomas A. Sellers, $\mathrm{PhD}^{39}$

Chavdar Slavov, DMSc ${ }^{40}$

Radka Kaneva, $\mathrm{PhD}^{41}$

Vanio Mitev, DSc ${ }^{41}$

Jyotsna Batra, $\mathrm{PhD}^{42}$

Judith A. Clements, $\mathrm{PhD}^{42}$

Amanda Spurdle, $\mathrm{PhD}^{43}$

Australian Prostate Cancer BioResource ${ }^{42,44}$

Manuel R. Teixeira, $\mathrm{PhD}^{45,46}$

Paula Paulo, $\mathrm{PhD}^{45}$

Sofia Maia, $\mathrm{PhD}^{45}$

Hardev Pandha, FRCP ${ }^{47}$

Agnieszka Michael, $\mathrm{PhD}^{47}$

Andrzej Kierzek, $\mathrm{PhD}^{47}$

David S. Karow, $\mathrm{MD}^{1,48}$

Ian G. Mills, PhD ${ }^{4,49}$

Ole A. Andreassen, $\mathrm{MD}^{4}$

Anders M. Dale, PhD $^{1,48,50}$

The PRACTICAL consortium*

\section{Affiliations}

${ }^{1}$ Center for Multimodal Imaging \& Genetics, University of California, San Diego, La Jolla, CA, USA

${ }^{2}$ Department of Radiation Medicine \& Applied Sciences, University of California, San

Diego, La Jolla, CA, USA

${ }^{3}$ Department of Cognitive Science, University of California, San Diego, La Jolla, CA, USA

${ }^{4}$ University of Oslo, Oslo, Norway

${ }^{5}$ Department of Surgery, University of California, San Diego, La Jolla, CA, USA

${ }^{6}$ The Institute of Cancer Research, London, SM2 5NG, UK

${ }^{7}$ Royal Marsden NHS Foundation Trust, London, SW3 6JJ, UK

${ }^{8}$ Centre for Cancer Genetic Epidemiology, Department of Public Health and Primary Care, University of Cambridge, Strangeways Research Laboratory, Worts Causeway, Cambridge CB1 8RN, UK

${ }^{9}$ Institute of Population Health, University of Manchester, Manchester, UK

${ }^{10}$ Warwick Medical School, University of Warwick, Coventry, UK

${ }^{11}$ Department of Medical Epidemiology and Biostatistics, Karolinska Institutet, Stockholm, Sweden

${ }^{12}$ Department of Molecular Medicine and Surgery, Karolinska Institutet, and Department of Urology, Karolinska University Hospital, Solna, 17176 Stockholm 
${ }^{13}$ Department of Medical Biochemistry and Genetics, Institute of Biomedicine, Kiinamyllynkatu 10, FI-20014 University of Turku; and Tyks Microbiology and Genetics, Department of Medical Genetics, Turku University Hospital

${ }^{14}$ BioMediTech, 30014 University of Tampere, Tampere, Finland

${ }^{15}$ Department of Medical Biochemistry and Genetics, Institute of Biomedicine, Kiinamyllynkatu 10, FI-20014 University of Turku, Finland

${ }^{16}$ Department of Urology, Tampere University Hospital and Medical School, University of Tampere, Finland

${ }^{17}$ Faculty of Health and Medical Sciences, University of Copenhagen, Denmark

${ }^{18}$ Department of Clinical Biochemistry, Herlev and Gentofte Hospital, Copenhagen University Hospital, Herlev, Denmark

${ }^{19}$ Department of Urology, Herlev and Gentofte Hospital, Copenhagen University Hospital, Herlev, Denmark

${ }^{20}$ Copenhagen Prostate Cancer Center, Department of Urology, Rigshospitalet, Copenhagen University Hospital, Copenhagen, Denmark

${ }^{21}$ Cancer Epidemiology, Nuffield Department of Population Health University of Oxford, Oxford, OX3 7LF, UK

${ }^{22}$ Nuffield Department of Surgery, University of Oxford, Room 6603, Level 6, John Radcliffe Hospital, Headley Way, Headington, Oxford, OX3 9DU, UK

${ }^{23}$ University of Cambridge, Department of Oncology, Box 279, Addenbrooke's Hospital, Hills Road Cambridge CB2 0QQ, UK

${ }^{24}$ School of Social and Community Medicine, University of Bristol, Canynge Hall, 39 Whatley Road, Bristol, BS8 2PS, UK

${ }^{25}$ Nuffield Department of Surgical Sciences, University of Oxford, Oxford, UK, Faculty of Medical Science, University of Oxford, John Radcliffe Hospital, Oxford, UK

${ }^{26}$ Centre for Cancer Genetic Epidemiology, Department of Oncology, University of Cambridge, Strangeways Research Laboratory, Worts Causeway, Cambridge, UK ${ }^{27}$ University College London, Department of Applied Health Research, 1-19 Torrington Place, London, WC1E 7HB

${ }^{28}$ Clinical Gerontology Unit, University of Cambridge, Cambridge UK

${ }^{29}$ Institute of Human Genetics, University Hospital of UIm, Ulm, Germany

${ }^{30}$ Department of Urology, Klinikum rechts der Isar der Technischen Universitaet Muenchen, Munich, Germany

${ }^{31}$ Division of Urologic Surgery, Brigham and Womens Hospital, Dana-Farber Cancer Institute, 75 Francis Street, Boston, MA 02115, USA

${ }^{32}$ International Hereditary Cancer Center, Department of Genetics and Pathology, Pomeranian Medical University, Szczecin, Poland

${ }^{33}$ Division of Genetic Epidemiology, Department of Medicine, University of Utah School of Medicine, Salt Lake City, Utah, USA

${ }^{34}$ George E. Wahlen Department of Veterans Affairs Medical Center, Salt Lake City, Utah, US

${ }^{35}$ Division of Clinical Epidemiology and Aging Research, German Cancer Research Center (DKFZ), Heidelberg, Germany 
${ }^{36}$ Division of Preventive Oncology, German Cancer Research Center (DKFZ) and National Center for Tumor Diseases (NCT), Heidelberg, Germany

${ }^{37}$ German Cancer Consortium (DKTK), German Cancer Research Center (DKFZ), Heidelberg, Germany

${ }^{38}$ Department of Cancer Epidemiology, Moffitt Cancer Center, 12902 Magnolia Drive, Tampa, FL 33612, USA

${ }^{39}$ Department of Biostatistics and Bioinformatics, Moffitt Cancer Center, 12902 Magnolia Drive, Tampa, FL 33612, USA

${ }^{40}$ Department of Urology and Alexandrovska University Hospital, Medical University, Sofia, Bulgaria

${ }^{41}$ Department of Medical Chemistry and Biochemistry, Molecular Medicine Center, Medical University, Sofia, 2 Zdrave Str., 1431 Sofia, Bulgaria

${ }^{42}$ Australian Prostate Cancer Research Centre-QId, Institute of Health and Biomedical Innovation and School of Biomedical Science, Queensland University of Technology, Brisbane, Australia

${ }^{43}$ Molecular Cancer Epidemiology Laboratory, Queensland Institute of Medical Research, Brisbane, Australia

${ }^{44}$ Australian Prostate Cancer BioResource, Institute of Health and Biomedical Innovation and School of Biomedical Science, Queensland University of Technology, Brisbane, Australia

${ }^{45}$ Department of Genetics, Portuguese Oncology Institute, Porto, Portugal

${ }^{46}$ Biomedical Sciences Institute (ICBAS), University of Porto, Porto, Portugal

${ }^{47}$ The University of Surrey, Guildford, Surrey, GU2 7XH

${ }^{48}$ Department of Radiology, University of California, San Diego, La Jolla, CA, USA

${ }^{49}$ Centre for Cancer Research and Cell Biology, Queens University Belfast, Belfast, UK

${ }^{50}$ Department of Neurosciences, University of California, San Diego, La Jolla, CA, USA

*Additional members from the Prostate Cancer Association Group to Investigate Cancer Associated Alterations in the Genome consortium (PRACTICAL, http://practical.ccge.medschl.cam.ac.uk/) provided in the Supplementary Material.

\section{Corresponding authors}

Tyler M. Seibert, MD, PhD

Center for Multimodal Imaging \& Genetics

Rm/Bldg: 4W102-B11 / Altman Building

UC San Diego Campus Direct

9500 Gilman Drive

La Jolla, CA 92093

United States

tseibert@ucsd.edu

(858) 255-1458

Anders M. Dale, PhD 


\author{
Center for Multimodal Imaging \& Genetics \\ Rm/Bldg: 4W228 / Altman Building \\ UC San Diego Campus Direct \\ 9500 Gilman Drive \\ La Jolla, CA 92093 \\ United States \\ amdale@ucsd.edu \\ (858) 822-6671
}




\title{
A genetic risk score to guide age-specific, personalized prostate cancer screening
}

\begin{abstract}
Background: Prostate-specific-antigen (PSA) screening resulted in reduced prostate cancer $(\mathrm{PCa})$ mortality in a large clinical trial, but due to a high false-positive rate, among other concerns, many guidelines do not endorse universal screening and instead recommend an individualized decision based on each patient's risk. Genetic risk may provide key information to guide the decisions of whether and at what age to screen an individual man for PCa.

Methods: Genotype, PCa status, and age from 34,444 men of European ancestry from the PRACTICAL consortium database were analyzed to select single-nucleotide polymorphisms (SNPs) associated with prostate cancer diagnosis. These SNPs were then incorporated into a survival analysis to estimate their effects on age at $\mathrm{PCa}$ diagnosis. The resulting polygenic hazard score (PHS) is an assessment of individual genetic risk. The final model was validated in an independent dataset comprised of 6,417 men with screening PSA and genotype data. PHS was calculated for these men to test for prediction of PCa-free survival. PHS was also combined with age-specific $\mathrm{PCa}$ incidence data from the U.S. population to generate a PCa-Risk (PCaR) age that relates a given man's risk to that of the population average. PHS and $\mathrm{PCaR}$ age were evaluated for prediction of positive predictive value (PPV) of PSA screening.
\end{abstract}

Findings: PHS calculated from 54 SNPs was very highly predictive of age at $\mathrm{PCa}$ diagnosis for men in the validation set $\left(p=10^{-53}\right)$. PPV of PSA screening varied from $0 \cdot 18$ to 0.52 for men with low and high genetic risk, respectively. PHS modulates PCa-free survival curves by an estimated 20 years between men in the $1^{\text {st }}$ or $99^{\text {th }}$ percentiles of genetic risk.

Interpretation: Polygenic hazard scores give personalized genetic risk estimates and can inform the decisions of whether and at what age to screen a man for PCa.

Funding: Department of Defense \#W81XWH-13-1-0391 


\section{Introduction}

Prostate cancer $(\mathrm{PCa})$ is a major health problem, with over one million new cases and over 300,000 prostate cancer deaths estimated worldwide in $2012^{1}$. An international, randomized, controlled trial showed that prostate-specific-antigen (PSA) screening resulted in a $20 \%$ reduction in PCa mortality by $20 \%{ }^{2}$. However, due to concerns over a high rate of false positives, in addition to aggressive treatment of initially indolent disease, many clinical guidelines do not endorse universal screening and instead stress the importance of taking into account individual patient risk factors to inform the decision of whether to screen ${ }^{3-5}$. The goal is to avoid unnecessary screening while still identifying high-risk men for whom screening and early PCa detection can reduce morbidity and mortality.

A patient's genetic predisposition could be critical to the decision of whether and when to offer him PCa screening. Genome-wide association studies (GWAS) have revealed genetic variants associated with increased risk of $\mathrm{PCa}^{6,7}$. These developments, combined with the recent accessibility of genotyping, provide an opportunity for genetic risk-informed cancer screening ${ }^{8}$. By combining risk information from an array of single nucleotide polymorphisms (SNPs), polygenic models can estimate an individual's genetic risk for developing the disease ${ }^{9}$. It remains unclear to what extent this predicted polygenic risk could improve clinical decisions such as whom to screen for PCa and at what age.

Here we use data from 34,444 men of European ancestry from the international PRACTICAL consortium (http://practical.ccge.medschl.cam.ac.uk/) to develop a polygenic hazard score (PHS) to predict age-related risk of developing prostate cancer. The PHS was then tested in data from an independent study (UK Protec $\mathrm{T}^{10}$ ) that included both genotype and PSA results, with the hypothesis that PHS would be an indicator of a patient's inherent genetic risk for developing prostate cancer at various ages in his lifetime.

\section{Methods}

\section{Participants}

Discovery Set: For PHS model development, genotype and age data were obtained from 21 studies of the PRACTICAL consortium (Table 1), representing 31,747 men $(18,868$ cases, 12,879 controls) of genotypic European ancestry. Age was either at PCa diagnosis or last follow-up (for controls. Genotyping was performed via a custom Illumina array (iCOGS), and quality control steps were applied as described previously ${ }^{6}$. 201,043 SNPs were available for analysis.

Validation Set: The model performance was examined in an independent study. The Validation Set comes from the ProtecT study, which screened 82,429 men with PSA testing and found 8,891 men with PSA greater than the specified threshold of $3.0 \mu \mathrm{g} / \mathrm{L}$ or higher, of whom 2,896 were diagnosed with $\mathrm{PCa}^{10}$. Among those individuals, we obtained genotype and age data for 6,411 men (1,583 cases, 4,828 controls). This data 
set was selected for validation because PSA results were also available for all participants at time of either diagnosis or interview.

\section{Polygenic hazard score (PHS)}

The PHS was developed previously as a parsimonious, survival-analysis model to predict the time to event outcome. It has been published elsewhere ${ }^{11}$. Because prostate cancer risk increases with age ${ }^{12}$ and anticipated age of developing prostate cancer is highly relevant to clinical management, we applied PHS for deriving both predicted absolute risk and potential age at PCa onset. In brief, a univariate trend test was applied to the entire Discovery Set (31,747 patients $\times 201,043$ SNPs) to assess association with case or control status. All SNPs with resulting $p$-values $<10^{-6}$ in the trend test were then entered in a forward, stepwise, greedy algorithm, to select the most predictive SNPs. In each step, logistic regression was used first to improve computational efficiency. SNPs were selected for the model only if they improved prediction of case-control status. After forward, stepwise selection, coefficients for selected SNPs were estimated using a Cox proportional hazard model to predict age at diagnosis with $\mathrm{PCa}$.

The polygenic hazard score (PHS) is defined as the vector product of a patient's genotype $\left(X_{i}\right)$ for the $n$ selected SNPs and the corresponding parameter estimates $\left(\beta_{i}\right)$ from the Cox proportional hazards regression.

$$
\text { PHS }=\sum_{i}^{n} X_{i} \beta_{i}
$$

To verify whether the PHS accurately predicts age of prostate cancer onset, the PHS was calculated for all patients in the Validation Set. These values were then tested as the sole predictive variable in a Cox proportional hazards regression model for age of diagnosis. Statistical significance was set at alpha of 0.01 .

\section{Estimate of absolute risk}

The population risk of prostate cancer was estimated using methods described previously $^{13}$. U.S. population risk data is reported by the American Cancer Society, with tables indicating the probability of developing PCa during specified age intervals, assuming the person is alive without $\mathrm{PCa}$ at the beginning of the interval ${ }^{12}$. Tables are constructed from the Surveillance, Epidemiology, and End Results (SEER) database. Data from four such tables were used, representing twelve years of SEER data in the period from 1995 to $2012^{12,14-16}$. Estimates were derived of age-specific incidence as follows. For example, for the period of 2010-2012, the probability of developing $\mathrm{PCa}$ from age 50 to 59 was $2 \cdot 1 \%^{12}$, so at a mean age of 54.5 years, we estimated the agespecific incidence as $2 \cdot 1 \% / 10$ years $=0.21 \% / y e a r$. This was done for each reported probability in the four publications cited. Age intervals prior to age 40 were excluded due to the very low incidence in the general population. For age intervals from 70 to death, 
the end of the interval was taken to be 83, given an average life expectancy of 14 years for a 70 -year-old $\operatorname{man}^{17}$. The age-specific incidence data points from all four publications were then fit to an exponential curve using linear regression in order to develop a continuous model of age-specific incidence in the U.S. SEER population.

\section{Examining impact of genetic risk on PSA screening}

To assess the clinical significance of PCa PHS, we looked at the positive predictive value (PPV) of PSA testing within the Validation Set, with clinical diagnosis (including biopsy result) as the gold standard. We posited that risk stratification with PHS would reflect the underlying incidence of $\mathrm{PCa}$ and therefore also affect the PPV of PSA testing.

In the Validation Set, 2,555 patients had positive PSA: 1,580 were then diagnosed with $\mathrm{PCa}$, while 975 were designated controls without PCa. Because genotype information was collected in more cases than controls, we matched the overall ProtecT control:case ratio $^{10}$ by taking a random sample of 471 cases with the 975 controls and calculating the positive predictive value of PSA testing without regard to PHS, as well as in subsets based on PHS percentile thresholds of $<20^{\text {th }},>50^{\text {th }},>80^{\text {th }}$, and $>95^{\text {th }}$. This process was repeated for a total of 1,000 random samples of 471 cases; mean and bootstrap estimate of the standard error were calculated for PPV in each PHS risk group.

To learn whether PHS impacts PPV within men of a given age category, we repeated the above PPV analysis for only Validation Set patients older than the median age of the group (60 years) and again for only those at or less than the median age.

The magnitude of PHS effect on expected age of onset was illustrated by calculating the PHS corresponding to percentiles among the young, healthy population within the Discovery Set: i.e., those controls with age $<70$ years. All percentiles reported in this manuscript refer to this population. Annualized incidence rate $\left(h_{\text {percentile }}\right)$ curves were calculated for each of various percentiles of the PHS from this population $(1,5,20,50$, $80,95,99)$ with the median PHS ( $\left.P H S_{\text {median }}\right)$ is taken as baseline:

$$
h_{\text {percentile }}(\text { age })=h_{S E E R}(\text { age }) e^{P H S_{\text {percentile }}-P H S_{\text {median }}}
$$

Application of an individualized PHS to screening decisions in the clinic would be facilitated by a readily interpretable translation of the PHS to terms familiar to the patient and physician. Thus, we introduce the "Prostate Cancer-Risk age" or PCaR age. An annualized incidence curve for the patient's PHS is generated just as was done for the population percentiles above, which gives an estimate of PCa risk. Then, for example, if a 50-year-old man has a PCa risk equivalent to that of the general population at age 60, his PCaR age is 60 .

A 95\% confidence interval is calculated for PCaR age by estimating the variance due to both genotypes in the Discovery Set and the SNP parameter estimates from the PHS model as follows (details in Supplementary Methods). 
The difference between PCaR age and true age (rounded to the nearest integer) is termed $\triangle$ age. The PCaR age and $\triangle$ age were calculated for every integer age between 40 and 95 years to assess whether $\triangle$ age changed over time. This was done for all PHS percentiles listed above, as well as $0 \cdot 1^{\text {th }}$ and $99 \cdot 9^{\text {th }}$.

In a common clinical situation, a patient of a given age may present to his physician to discuss screening. To illustrate how PHS might influence this discussion, we identified the subset of Validation patients at approximately the median age, 60 years (57-63), to represent a typical patient. From this subset of 945 men around 60 years old, three groups were created: those whose PCaR age was also within the 57-63 interval, those with PCaR age $<57$, and those with PCaR age $>63$. We then calculated the PPV of PSA for these three groups using the same approach as before.

\section{Results}

Of the 201,043 SNPs included in the data set, 2,415 were associated with increased risk of $\mathrm{PCa}$ in the trend test, with $p<10^{-6}$. The stepwise regression framework then identified 54 of these SNPs that were incorporated into the Cox proportional hazards model (Supplementary Table S1). The 54 SNP parameter estimates (for the hazard of developing $\mathrm{PCa}$ ) are combined with individual genotype to generate a polygenic hazard score. Kaplan-Meier curves indicate that the assumption of proportional hazards was reasonable in the final model (Figure 1).

In the independent Validation Set from the ProtecT study, a Cox proportional hazards model showed that PHS significantly predicted age of prostate cancer onset $(z=15 \cdot 4$, $p=10^{-53}$.

Positive predictive value of PSA testing in the Validation Set is plotted in Figure 2. PPV was lower among patients with a low PHS, and higher among patients with progressively higher PHS. Patients with PHS $<20^{\text {th }}$ percentile had PPV 0.18 , while those with PHS $>95^{\text {th }}$ percentile had PPV 0.52. Within the $\leq 60$ and $>60$ age groups, PHS stratification still resulted in notable changes in the PPV of PSA testing (Figure 3).

Absolute risk of PCa for the general U.S. population was estimated with linear regression using data from the SEER database from 1995 to $2012^{12,14-16}$. The resulting model for hazard rate $\left(h_{S E E R}\right)$ had $R^{2}=0.88$ and $p=10^{-5}$ (Figure 4):

$$
h_{S E E R}(\text { age })=0.0700 e^{0.0753(\text { age }-40)}
$$

Annualized incidence and survival curves corresponding to PHS percentiles (among controls $<70$ years old) are shown in Figure 5. A table of prostate cancer-risk (PCaR) ages for various PHS levels demonstrates shows that the expected age of PCa onset is modulated by 20 years between the $1^{\text {st }}$ and $99^{\text {th }}$ PHS percentiles and by nearly 50 years between the $0 \cdot 1^{\text {th }}$ and $99 \cdot 9^{\text {th }}$ percentiles (Table 2).

Qualitatively, the curves in Figure 5 appear to maintain relatively consistent horizontal shifts relative to their neighbors over the age range studied. Quantitatively, this is confirmed by $\Delta$ age, which remained the same for each PHS percentile across a true 
age range of 40 to 95 . Thus, $\Delta$ age was taken to be approximately constant for each PHS percentile and is reported in Table 2.

The PPV of PSA testing for Validation Set patients approximately 60 years of age (5763 ) is shown in Figure 6. PPV was lower for those with PCaR age $<57$ and higher for those with PCaR age $>63$.

\section{Discussion}

Genetic information may be the ideal guide for deciding whether an individual patient needs prostate cancer screening ${ }^{8}$. The polygenic hazard score described here represents a personalized genetic assessment of a patient's age-related prostate cancer risk that can inform both whether and when to order screening tests. In a survival analysis using patients from an independent clinical trial, PHS was a remarkably strong predictor $\left(p=10^{-53}\right)$ of age at $\mathrm{PCa}$ diagnosis. Furthermore, riskstratification with PHS had considerable impact on the positive predictive value of PSA testing. For example, among patients with elevated PSA, only $18 \%$ of those with low PHS were true positives, whereas over half of those with high PHS had prostate cancer (Figure 3). As PHS is representative of a man's fixed genetic risk, it can be calculated long before onset of PCa and substantially inform the decision of whether he should undergo PCa screening.

Because PCa incidence is highly dependent on age ${ }^{12}$, one must decide not only whether to screen but also at what age to consider it. Prostate Cancer-Risk (PCaR) age incorporates a patient's true age and genetic risk to give an adjusted age that relates his current risk of $\mathrm{PCa}$ to that of the age-specific population average. For example, a physician who normally discusses the risks and benefits of PSA screening with her patients starting at age 50 could shift the timing of that conversation for each patient according to his PCaR age. Using the U.S. SEER database as the population average, we show here that PHS modulates PCa-free survival curves by 20 years between the $1^{\text {st }}$ and $99^{\text {th }}$ percentiles (Figure 5). The comparison is even more extreme for PHS percentiles 0.1 and 99.9 (Table 2), with some men not reaching the risk of a typical 50 year-old until age 85, while others reach that risk at age 37 . With nearly 40 million men aged $50-70$ years in the U.S. alone ${ }^{18}$, almost 80,000 would presumably fall into one of these extreme PHS categories.

To illustrate the usefulness of PCaR age, we consider a common clinical scenario where a man in clinic asks about screening. We assume his age is 60 , the median for the ProtecT cohort. Figure 6 shows results for ProtecT patients approximately 60 (5763) years old and suggests that if this 60-year-old man's PCaR age is close to his true age (i.e., still 57-63), the PPV of a PSA test for him now is $29 \%$, close to the average for PSA screening in general ${ }^{3}$. If his PCaR age is under 57, the PPV drops to around $13 \%$, and he might be reassured that a PSA test is not necessary. On the other hand, if his $\mathrm{PCaR}$ age is over 63, the PPV is approaching $50 \%$, which might make PSA more informative. 
PSA screening is controversial, but most guidelines recommend individualized discussion between physicians and patients ${ }^{12,14-16}$. PHS affords a quantitative and understandable way to evaluate individual risk that could prove pivotal in these discussions. The results here suggest PHS can be used to identify a large percentage of men for whom forgoing or delaying PSA screening makes sense. At the same time, $\mathrm{PHS}$ can also identify men with a high risk of developing $\mathrm{PCa}$ at a young age and who therefore may benefit from early detection through PSA screening.

Another concern with PSA screening is overtreatment of indolent disease. Genetic prediction of aggressive $\mathrm{PCa}$ alone has proven elusive ${ }^{19}$, and the problem is compounded by the propensity of initially low-risk tumors to progress ${ }^{20,21}$. Datasets describing the initial tumor characteristics are not enough-tumors that develop aggressive features over time must also be identified if aggressive-PCa-only predictors are to be effective. Active surveillance is one answer to overtreatment that avoids upfront aggressive treatment but still allows intervention if the tumor progresses. Indeed, most tumors will eventually require treatment ${ }^{21,22}$, and earlier treatment prevents development of metastatic disease ${ }^{22}$. Hence, avoiding screening altogether in patients who may develop PCa at a young age does carry risk of considerable morbidity.

While PHS was applied here to PSA screening, PHS itself is not specific to PSA testing. Rather, the PHS is predictive of patients' underlying risk of $\mathrm{PCa}$ at a given age and therefore relates to pre-test probability - and, by extension, positive predictive valuewithin any screening strategy that might be adopted.

Cost effectiveness is a prominent concern in all discussions of healthcare policy. PHS has the potential to improve screening efficiency while also reducing overall costs. PHS need only be calculated once and is valid for a lifetime. The genotyping chip assay can be run for costs in the range of that for single-gene testing (e.g., BRCA mutation), informs multiple disease ${ }^{23}$, and a saliva sample suffices. PSA screening and subsequent biopsies could thus be limited to those men at higher risk, while delaying or forgoing screening in those whose genetic makeup confers a low risk.

Prior studies have used GWAS-associated polymorphisms to predict risk of PCa using a case/control design ${ }^{24-26}$. However, epidemiologic data show that PCa risk is not a simple dichotomy of cases and controls, but rather is highly dependent on increasing age. Therefore, we opted for a survival analysis approach optimized for genetic prediction of age of PCa onset. The PHS can then be used in clinical decisions, where age plays a critical role. If a man has a high risk of developing prostate cancer at age 95, this is a very different clinical situation from a man at high risk at age 55.

There are several limitations to this study. It is inherently a retrospective analysis, but the Discovery Set data come from large studies carried out in multiple institutions and nations; the Validation Set, too, comes from an independent, large, prospective trial. The absolute risk models shown in Figure 5 are only as accurate as the population data upon which they are based, which reflect diagnosed prostate cancer in the U.S. It is important to note, though, that PHS is a measure of hazard and therefore could be readily applied to other population incidence curves to estimate absolute risk in those 
populations. The age range of the Validation Set is limited to only 50-70 years; fortunately, this includes the age where screening is believed to have the most benefit ${ }^{12,14-16}$. Finally, race in this PHS model is limited to European ancestry. Validation of PHS in other racial groups - and, if necessary, custom models for each-is needed; our group plans to investigate this important question.

In conclusion, we describe here the development of a new polygenic hazard score for personalized genetic assessment of individual, age-associated prostate cancer risk. This score has been validated in an independent data set, demonstrating accurate prediction of prostate cancer onset. Moreover, PHS is shown to predict the utility of PSA testing for an individual patient and can guide the decision of whether and when to screen for prostate cancer. 


\section{References}

1 Torre LA, Bray F, Siegel RL, Ferlay J, Lortet-Tieulent J, Jemal A. Global cancer statistics, 2012. CA Cancer J Clin 2015; 65: 87-108.

2 Schröder FH, Hugosson J, Roobol MJ, et al. Screening and Prostate-Cancer Mortality in a Randomized European Study. N Engl J Med 2009; 360: 1320-8.

3 Wolf AMD, Wender RC, Etzioni RB, et al. American Cancer Society Guideline for the Early Detection of Prostate Cancer: Update 2010. CA Cancer J Clin 2010; 60: 70-98.

4 Horwich A, Hugosson J, de Reijke T, et al. Prostate cancer: ESMO Consensus Conference Guidelines 2012. Ann Oncol Off J Eur Soc Med Oncol ESMO 2013; 24: 1141-62.

5 Qaseem A, Barry MJ, Denberg TD, Owens DK, Shekelle P, Clinical Guidelines Committee of the American College of Physicians. Screening for prostate cancer: a guidance statement from the Clinical Guidelines Committee of the American College of Physicians. Ann Intern Med 2013; 158: 761-9.

6 Eeles RA, Olama AAA, Benlloch S, et al. Identification of 23 new prostate cancer susceptibility loci using the iCOGS custom genotyping array. Nat Genet 2013; 45: 385-91.

7 Goh CL, Schumacher FR, Easton D, et al. Genetic variants associated with predisposition to prostate cancer and potential clinical implications. J Intern Med 2012; 271: 353-65.

8 Witte JS. Personalized Prostate Cancer Screening: Improving PSA Tests with Genomic Information. Sci Transl Med 2010; 2: 62ps55-62ps55.

9 Chatterjee N, Shi J, García-Closas M. Developing and evaluating polygenic risk prediction models for stratified disease prevention. Nat Rev Genet 2016; advance online publication. DOI:10.1038/nrg.2016.27.

10Lane JA, Donovan JL, Davis M, et al. Active monitoring, radical prostatectomy, or radiotherapy for localised prostate cancer: study design and diagnostic and baseline results of the ProtecT randomised phase 3 trial. Lancet Oncol 2014; 15: 1109-18.

11 Desikan RS, Fan CC, Wang Y, et al. Personalized genetic assessment of age associated Alzheimers disease risk. bioRxiv 2016; : 074864.

12 Siegel RL, Miller KD, Jemal A. Cancer statistics, 2016. CA Cancer J Clin 2016; 66: 7-30.

13Brookmeyer R, Gray S, Kawas C. Projections of Alzheimer's disease in the United States and the public health impact of delaying disease onset. Am J Public Health 1998; 88: 1337-42.

14Siegel R, Ward E, Brawley O, Jemal A. Cancer statistics, 2011. CA Cancer J Clin 2011; 61: 212-36.

15 Jemal A, Siegel R, Ward E, et al. Cancer Statistics, 2006. CA Cancer J Clin 2006; 56: 10630. 
16Greenlee RT, Hill-Harmon MB, Murray T, Thun M. Cancer Statistics, 2001. CA Cancer J Clin 2001; 51: 15-36.

17 Arias E. United States life tables, 2008. Hyattsville, MD: National Center for Health Statistics, 2012 http://www.cdc.gov/nchs/data/nvsr/nvsr61/nvsr61_03.pdf.

18United States Population by Age and Sex. United States Census Bureau, 2016 http://www.census.gov/popclock/data_tables.php?component=pyramid (accessed Sept 15, 2016).

19Eeles R, Goh C, Castro E, et al. The genetic epidemiology of prostate cancer and its clinical implications. Nat Rev Urol 2014; 11: 18-31.

20Pashayan N, Pharoah P, Neal DE, et al. PSA-detected prostate cancer and the potential for dedifferentiation - estimating the proportion capable of progression. Int J Cancer 2011; 128: $1462-70$.

21 Fleshner NE, Lucia MS, Egerdie B, et al. Dutasteride in localised prostate cancer management: the REDEEM randomised, double-blind, placebo-controlled trial. The Lancet 2012; 379: 1103-11.

22 Hamdy FC, Donovan JL, Lane JA, et al. 10-Year Outcomes after Monitoring, Surgery, or Radiotherapy for Localized Prostate Cancer. N Engl J Med 2016; 0: null.

23 Michailidou K, Hall P, Gonzalez-Neira A, et al. Large-scale genotyping identifies 41 new loci associated with breast cancer risk. Nat Genet 2013; 45: 353-61.

24Amin Al Olama A, Benlloch S, Antoniou AC, et al. Risk Analysis of Prostate Cancer in PRACTICAL, a Multinational Consortium, Using 25 Known Prostate Cancer Susceptibility Loci. Cancer Epidemiol Biomarkers Prev 2015; 24: 1121-9.

25 Pashayan N, Duffy SW, Neal DE, et al. Implications of polygenic risk-stratified screening for prostate cancer on overdiagnosis. Genet Med 2015; 17: 789-95.

26Mikropoulos C, Goh C, Leongamornlert D, Kote-Jarai Z, Eeles R. Translating genetic risk factors for prostate cancer to the clinic: 2013 and beyond. Future Oncol 2014; 10: 1679-94. 


\section{Declaration of Interests}

TMS reports honoraria from WebMD, Inc. for educational content, as well as a research grant from Varian Medical Systems, all outside the present study. ASK reports advisory board memberships for Sanofi-Aventis, Dendreon, and Profound, all outside the present study. AK reports paid work for Certara Quantitative Systems Pharmacology and consultancy contracts for pharmaceutical companies, all outside the present study. DSK reports paid work for Human Longevity, Inc., outside the present study. OAA reports research grants from KG Jebsen Stiftelsen, Research Council of Norway, and South East Norway Health Authority during the conduct of the study; additionally, he has a patent application (\# U.S. 20150356243) pending. AMD is a founder, equity holder, and advisory board member for CorTechs Labs, Inc.; advisory board member of Human Longevity, Inc.; recipient of non-financial research support from General Electric Healthcare; all of these disclosures are outside the present work. AMD also has a pending patent application assigned to UC San Diego (\# U.S. 20150356243). Other authors have no disclosures beyond the funding sources for the study.

\section{Author Contributions}

TMS, CCF, VZ, DSK, IGM, OAA, and AMD designed the study. RAE, DFE, ZSKJ, AAAO, SBG, KM, HG, FW, MA, JS, CSi, TLJT, BGN, SFN, MW, RB, MAR, PI, TJK, RCT, DEN, JLD, FCH, PPh, NP, KTK, CM, WV, ML, KH, ASK, CC, DW, WK, LCA, HB, KC, KUS, JYP, TAS, CSI, RKan, VM, JB, JAC, AS, APCBR, MRT, PPa, SM, HP, AM, and AK collected the data. TMS, CCF, IGM, OAA, and AMD performed the literature search. TMS, CCF, YW, VZ, RKar, DSK, and AMD performed the data analysis. TMS, CCF, RKar, JKP, DSK, OAA, and AMD interpreted the data. TMS, RKar, JKP, DSK, OAA, and AMD created the figures. TMS, CCF, OAA, and AMD wrote the manuscript. All authors reviewed the manuscript, added appropriate revisions, agreed to submission for publication, and approved the final version.

\section{Funding Source}

This study was funded in part by a grant from the United States Department of Defense (\#W81XWH-13-1-0391). Funding for the PRACTICAL consortium member studies is detailed in the supplementary material. No funding source had any role in the design, collection, analysis, interpretation, or writing of this study and associated manuscript, nor in the decision to submit it for publication. 


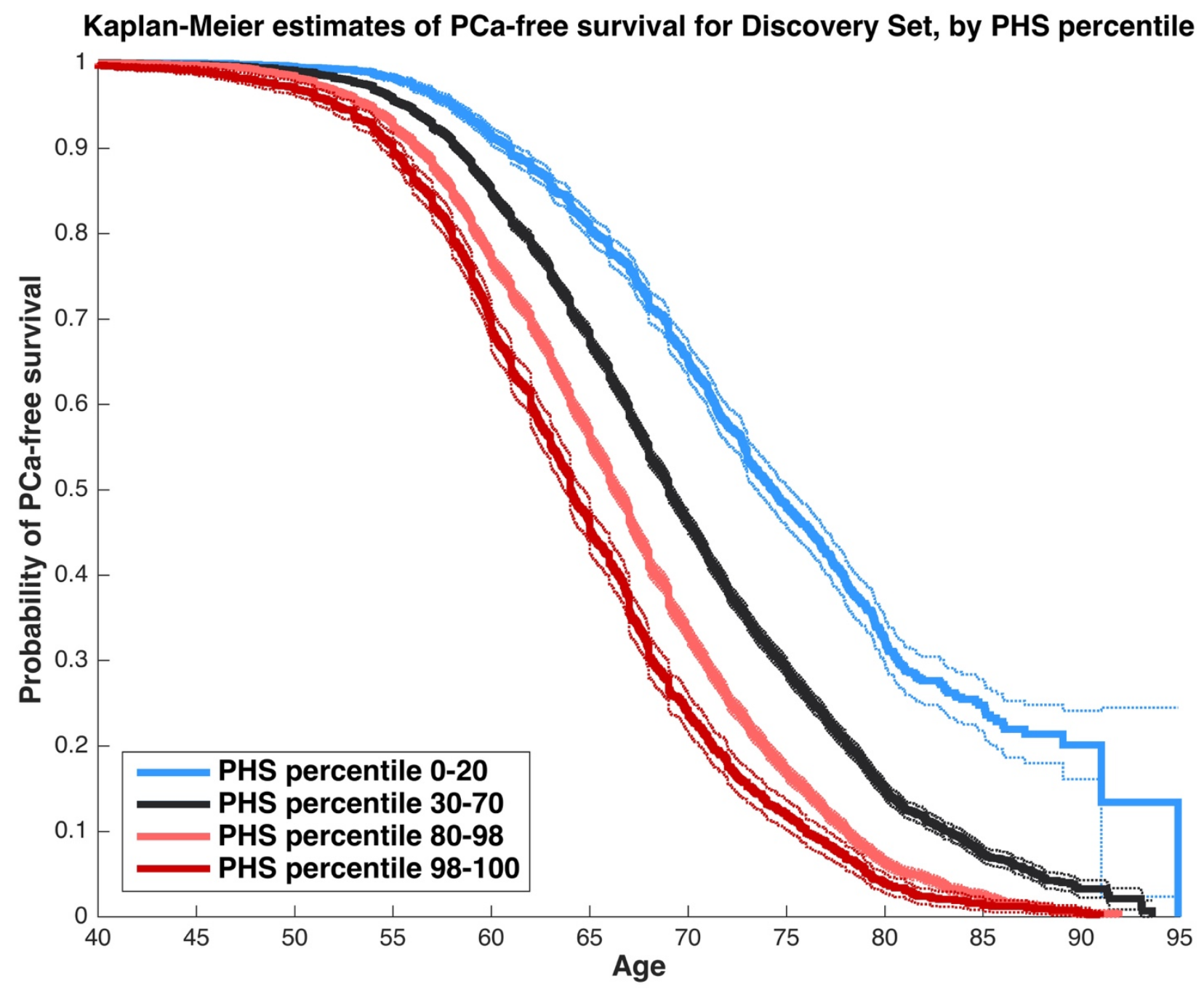

Figure 1: Kaplan-Meier estimates of prostate cancer-free survival for patients in the Discovery Set, grouped by PHS percentile ranges (as shown in the legend). PHS percentiles are in reference to the distribution of PHS within the 11,190 controls in the Discovery Set who were under 70 years old. Dotted lines are $95 \%$ confidence intervals for the corresponding curves. Time of "failure" is age at prostate cancer diagnosis. Controls were censored at age of observation. These curves demonstrate that the proportional hazards assumption holds for this PHS model. 


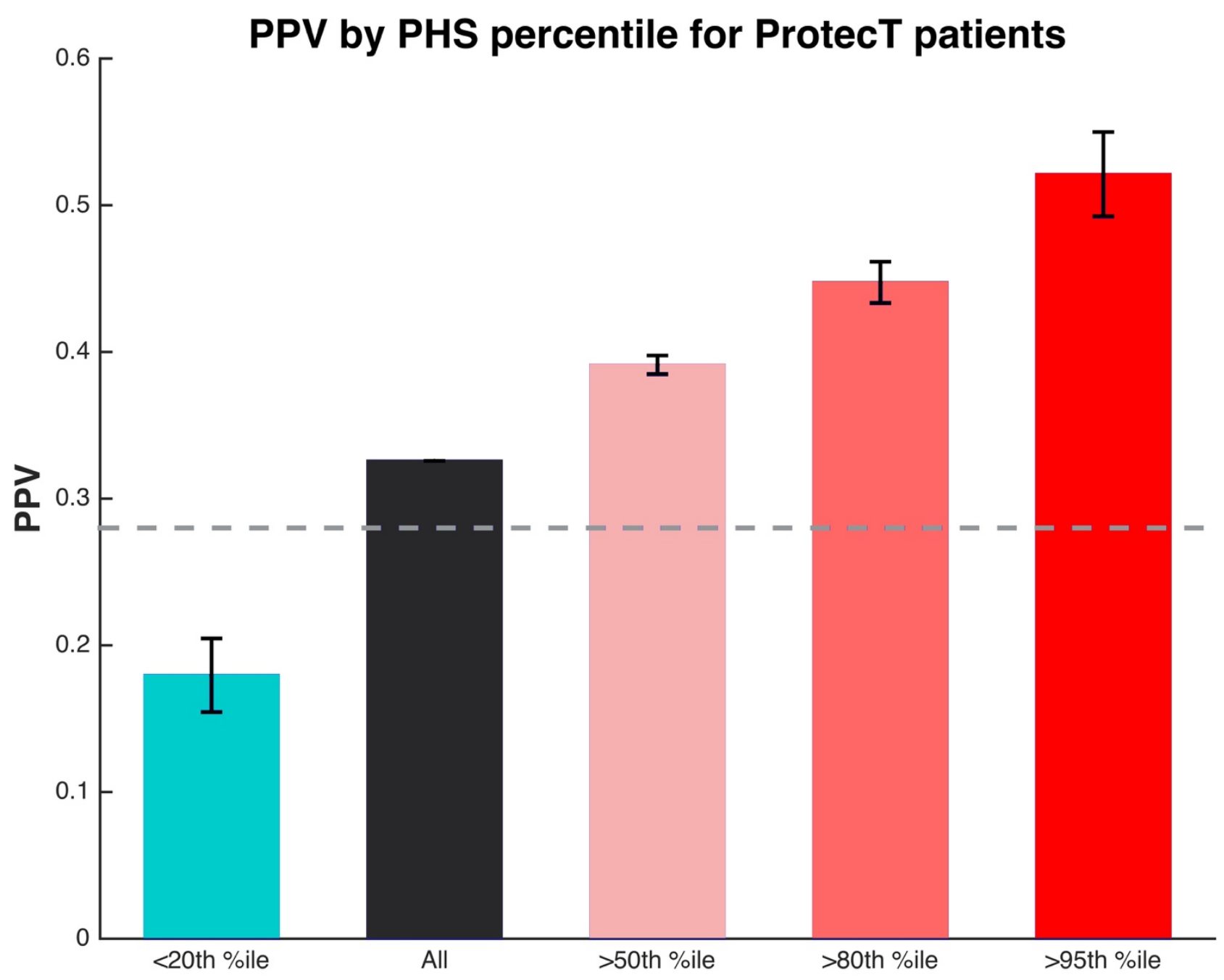

Figure 2: Positive predictive value (PPV) of PSA testing by PHS percentile thresholds for patients in the Validation Set. Percentiles refer to the PHS distribution among young controls in the Discovery Set. Error bars are the bootstrap estimate of the standard error for random samples of cases in the Validation Set (see Methods). For reference, the expected PPV for PSA testing at this threshold is displayed as a gray, dashed line, based on a pooled analysis ${ }^{3}$. 

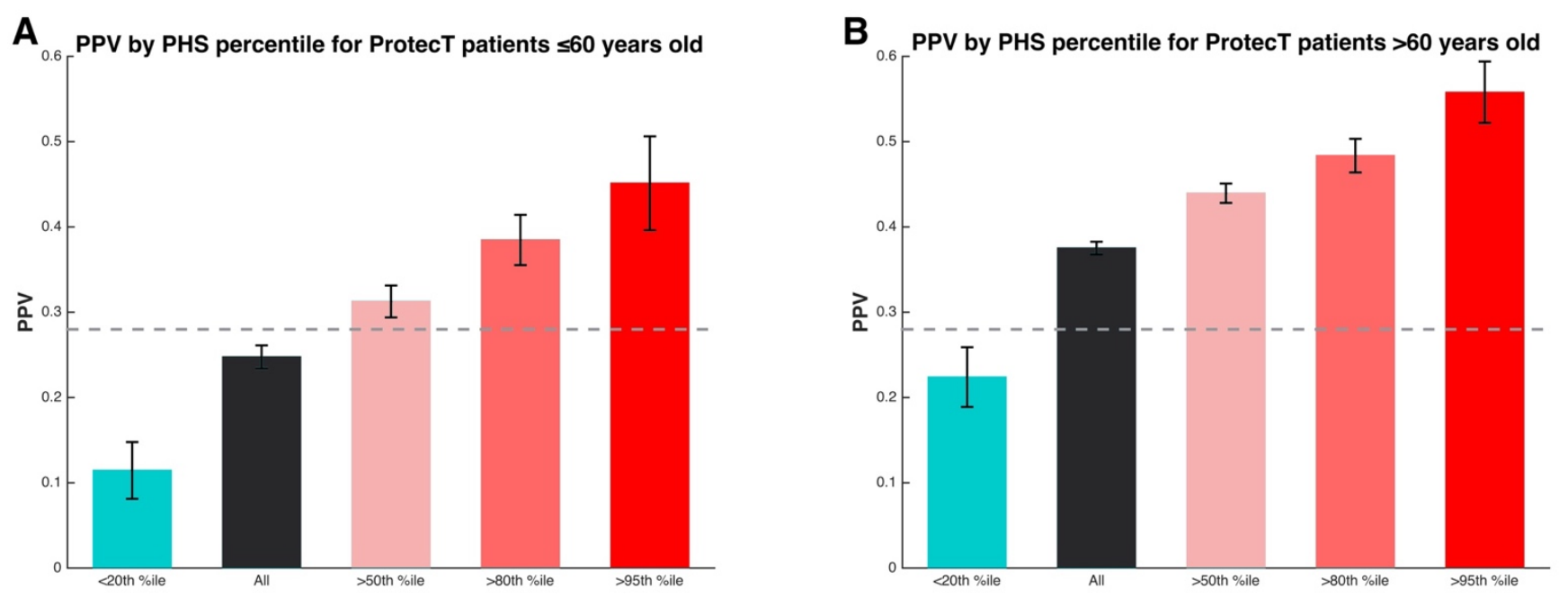

Figure 3: Positive predictive value (PPV) of PSA testing by PHS percentile thresholds for patients in the Validation Set, split by age group. (A) shows results for patients at or below the median age (60), and (B) shows results for patients older than the median age. For both panels: percentiles refer to the PHS distribution among young controls in the Discovery Set. Error bars are the bootstrap estimate of the standard error for random samples of cases in the Validation Set (see Methods). For reference, the expected PPV for PSA testing at this threshold is displayed as a gray, dashed line, based on a pooled analysis ${ }^{3}$. 


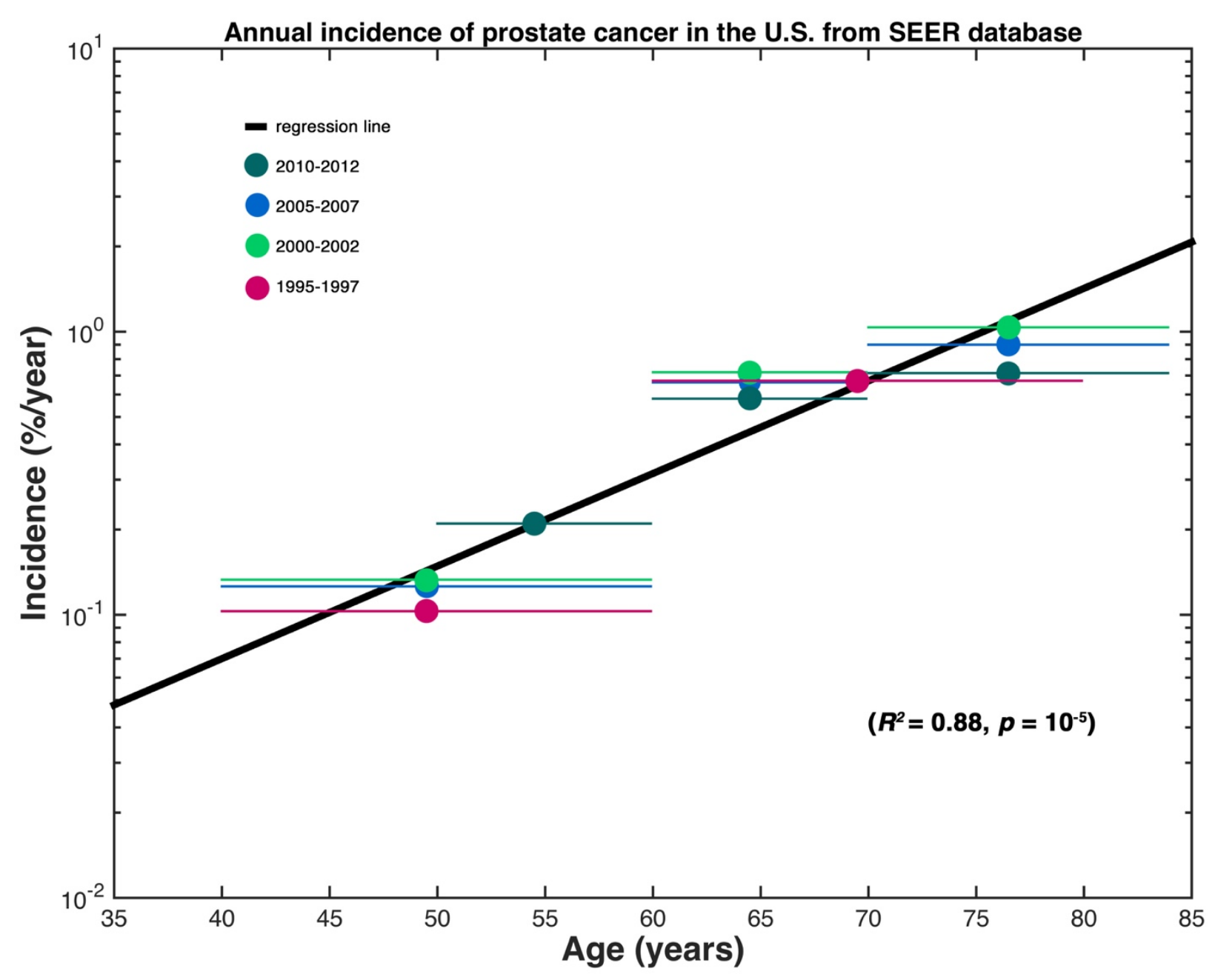

Figure 4: Dots represent the annual, age-specific incidence estimated from each age range (shown as horizontal lines of matching color) from U.S. population data in the SEER database. Dot color corresponds to the years the data were collected from, as shown in the legend. The black line is the result of linear regression for an exponential curve to give a continuous model of age-specific incidence in the U.S. population. 
A

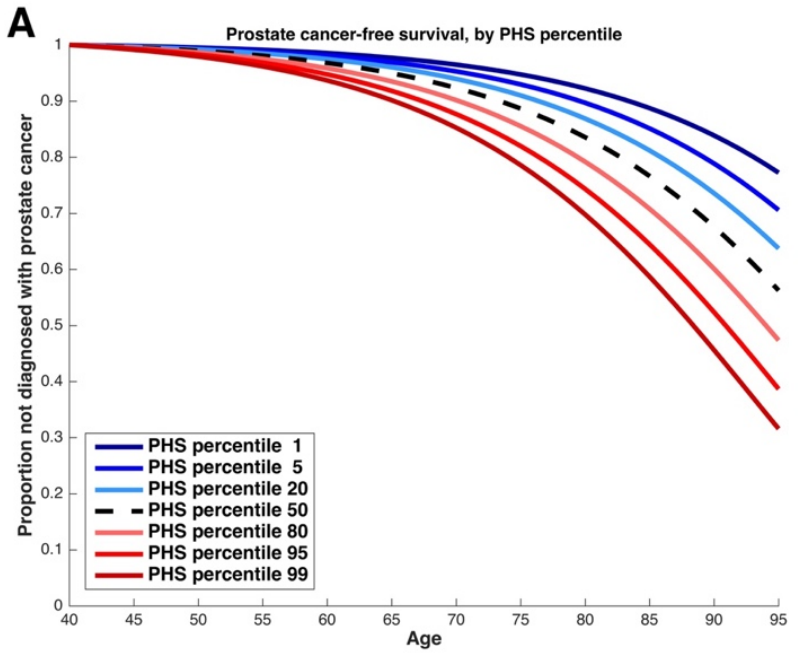

B

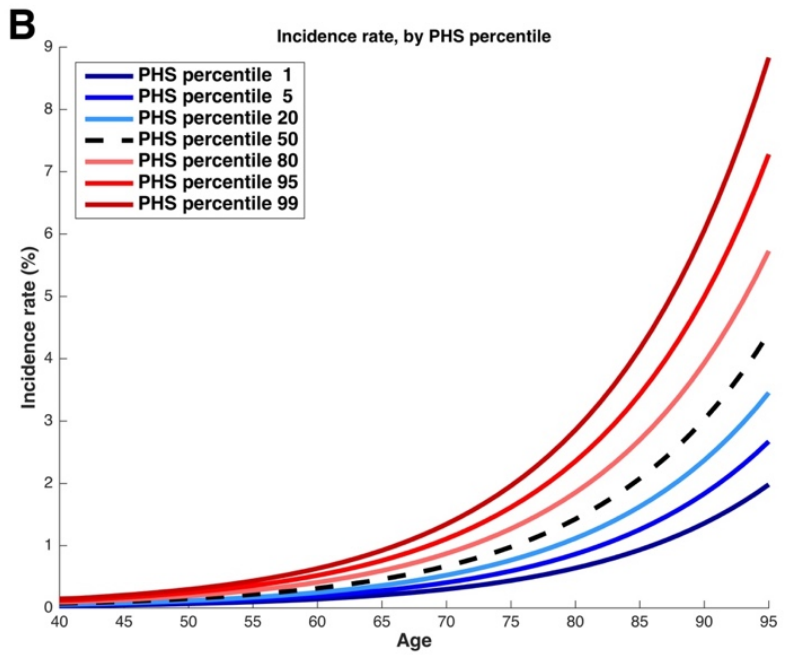

Figure 5: (A) Prostate cancer-free survival curves derived from PHS hazards, with U.S. population data taken as the median risk. PHS percentiles are in reference to the distribution of PHS within the 11,190 controls in the Discovery Set less than 70 years old. Blue lines represent genetic risk lower than the median, and red lines represent genetic risk higher than the median. $(B)$ Incidence rate curves by age for the same risk levels as in $(A)$. 


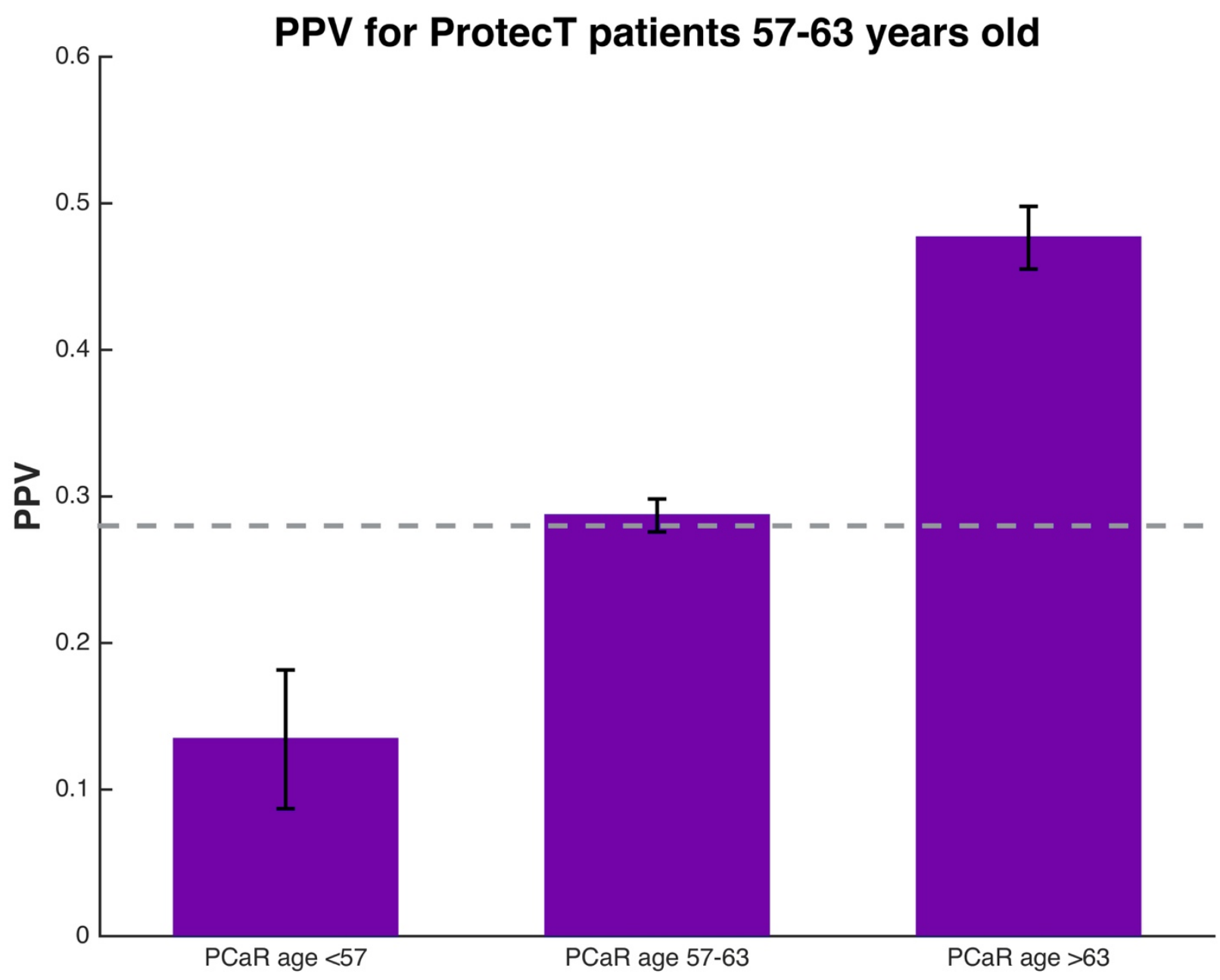

Figure 6: Application of Prostate Cancer-Risk (PCaR) age to the question of whether to screen a 60-year-old man. PCaR age is his true age adjusted by PHS level. For example, if his PCaR age is 71 years, his estimated risk matches that of a typical 71year-old. Alternatively, a PCaR age of 52 would mean his PCa risk is similar to a typical 52 -year-old. The bar plot shows results for all men from the Validation Set with age approximately 60 years (57-63), grouped by their calculated PCaR age: $<57,57-63$, or $>63$. The positive predictive value (PPV) of PSA testing is shown for these groups. Error bars are the bootstrap estimate of the standard error. For reference, the expected PPV for PSA testing is displayed as a gray, dashed line, based on a pooled analysis ${ }^{3}$. $~ 68 \%$ of men had PCaR age 57-63 and PPV close to the expected population value. 26\% and $\sim 7 \%$ of men were in the higher and lower groups, respectively. 
Table 1: Study names and participant numbers

\begin{tabular}{|c|c|c|c|}
\hline & Cases & Controls & Total \\
\hline \multicolumn{4}{|l|}{ Discovery Set } \\
\hline CAPS & 1,022 & 587 & 1,609 \\
\hline CPCS1 & 848 & 2,748 & 3,596 \\
\hline CPCS2 & 264 & 1,001 & 1,265 \\
\hline EPIC & 722 & 1,078 & 1,800 \\
\hline EPIC-Norfolk & 484 & 917 & 1,401 \\
\hline ESTHER & 313 & 318 & 631 \\
\hline IPO-Porto & 183 & 66 & 249 \\
\hline MAYO & 767 & 488 & 1,255 \\
\hline MOFFITT & 414 & 100 & 514 \\
\hline PCMUS & 149 & 140 & 289 \\
\hline PPF-UNIS & 245 & 188 & 433 \\
\hline Poland & 438 & 358 & 796 \\
\hline ProMPT & 166 & 2 & 168 \\
\hline QLD & 184 & 87 & 271 \\
\hline SEARCH & 1,371 & 1,239 & 2,610 \\
\hline STHM1 & 1,717 & 1,886 & 3,603 \\
\hline TAMPERE & 2,736 & 2,413 & 5,149 \\
\hline UKGPCS & 3,957 & 511 & 4,468 \\
\hline ULM & 599 & 354 & 953 \\
\hline UTAH & 439 & 244 & 683 \\
\hline WUGS & 4 & 0 & 4 \\
\hline All & 17,022 & 14,725 & 31,747 \\
\hline \multicolumn{4}{|l|}{ Validation Set } \\
\hline Protec $^{a}$ & 1,583 & 4,828 & 6,411 \\
\hline
\end{tabular}

${ }^{a}$ Includes the 31 cases and 3,364 controls who participated in both ProtecT and UKGPCS 
Table 2: Prostate cancer-risk (PCaR) age

\begin{tabular}{|c|c|c|c|}
\hline PHS percentile ${ }^{a}$ & $\begin{array}{c}\Delta \mathrm{Age}^{b} \\
{[95 \% \mathrm{Cl}]}\end{array}$ & $\begin{array}{l}\text { PCaR age when } \\
\text { true age is } 50 \text { years }\end{array}$ & $\begin{array}{l}\text { Age when risk is that of } \\
\text { typical } 50 \text {-year-old }\end{array}$ \\
\hline 0.1 & $\begin{array}{c}-35 \\
{[-35,-19]}\end{array}$ & $\begin{array}{c}15 \\
{[15,31]}\end{array}$ & $\begin{array}{c}85 \\
{[69,101]}\end{array}$ \\
\hline 1 & $\begin{array}{c}-11 \\
{[-15,-6]}\end{array}$ & $\begin{array}{c}39 \\
{[35,44]}\end{array}$ & $\begin{array}{c}61 \\
{[56,65]}\end{array}$ \\
\hline 5 & $\begin{array}{c}-7 \\
{[-11,-2]}\end{array}$ & $\begin{array}{c}43 \\
{[39,48]}\end{array}$ & $\begin{array}{c}57 \\
{[52,60]}\end{array}$ \\
\hline 20 & $\begin{array}{c}-3 \\
{[-7,1]}\end{array}$ & $\begin{array}{c}47 \\
{[43,51]}\end{array}$ & $\begin{array}{c}53 \\
{[49,57]}\end{array}$ \\
\hline 50 & $\begin{array}{c}0 \\
{[-4,4]}\end{array}$ & $\begin{array}{c}50 \\
{[46,54]}\end{array}$ & $\begin{array}{c}50 \\
{[46,54]}\end{array}$ \\
\hline 80 & $\begin{array}{c}\mathbf{3} \\
{[0,7]}\end{array}$ & $\begin{array}{c}53 \\
{[50,57]}\end{array}$ & $\begin{array}{c}47 \\
{[43,50]}\end{array}$ \\
\hline 95 & $\begin{array}{c}7 \\
{[3,10]}\end{array}$ & $\begin{array}{c}57 \\
{[53,60]}\end{array}$ & $\begin{array}{c}43 \\
{[40,47]}\end{array}$ \\
\hline 99 & $\begin{array}{c}\mathbf{9} \\
{[5,13]}\end{array}$ & $\begin{array}{c}\mathbf{5 9} \\
{[55,63]}\end{array}$ & $\begin{array}{c}\mathbf{4 1} \\
{[37,45]}\end{array}$ \\
\hline 99.9 & $\begin{array}{c}13 \\
{[9,16]}\end{array}$ & $\begin{array}{c}63 \\
{[59,66]}\end{array}$ & $\begin{array}{c}37 \\
{[34,41]}\end{array}$ \\
\hline
\end{tabular}

${ }^{a} \mathrm{PHS}$ percentile among young ( $<70$ years old) controls within Discovery Set.

${ }^{b} \Delta$ Age $=\mathrm{PCaR}$ age - true age.

${ }^{c}$ Risk of typical 50-year-old defined as overall population incidence at age 50. 


\section{Research in Context}

\section{Evidence before this study}

Prostate cancer ( $\mathrm{PCa}$ ) screening with prostate-specific-antigen (PSA) testing can lead to early detection of $\mathrm{PCa}$ and allow for curative treatment, but universal screening also has considerable disadvantages for men who may never develop life-threatening disease. Whom to screen and at what age to do so remain unclear.

Genetic studies have shown that single-nucleotide polymorphisms (SNPs) have modest predictive value for PCa risk, but that a combination of genotype information from multiple SNPs can give a more robust PCa risk prediction. The practical, clinical utility of SNP-based prediction of expected age of PCa onset is not well understood.

\section{Added value of this study}

This study presents and validates a novel polygenic hazard score that is an indicator of PCa-free survival. This polygenic hazard score (PHS) offers a relatively inexpensive assessment of an individual man's age-specific PCa risk.

\section{Implications of all the available evidence}

SNP-based polygenic hazard scores can provide objective, readily interpretable information to guide the decision of whether a given patient might benefit from $\mathrm{PCa}$ screening at each age in his life 